\title{
Custom setup for organic crystal growth by vapor deposition
}

\author{
Georgii Bogdanov, Sergei Rigin, Gil Gallegos, Tatiana V. Timofeeva
}

Departments of Chemistry and Computer Science, New Mexico Highlands University, Las Vegas, NM, USA

Organic electronics and optoelectronics have recently drawn significant attention because they are easy to manufacture, light weight by nature, structurally flexible and possess many other desirable properties which are difficult to achieve using inorganic electronic materials. In recent years much attention was paid to materials built of two or more components. For example, donor and acceptor molecules were given much focus. For such materials, strong correlation between their internal structure and properties usually exists. Our group is focused on creation of two-component organic or organic-inorganic crystals with the potential application as semiconductors and light emitting diodes.

There are several ways to obtain crystals with new crystalline structures. Our approach will afford synthesis of crystals utilizing novel growth techniques. The most common way is growth from solution. Presently, schemes that utilize novel vapor deposition growth are on the horizon. Thus, this new method will enable growth of low soluble materials with improved crystal structure. This approach is based on the physical phenomenon known as sublimation.

Our research goal was to create an improved crystal growth test bed utilizing the methods discussed above. Our method allows us to grow crystals with much higher purity and larger size than was possible utilizing the more traditional solution growth approach. Based on publications [1-3] and using recommendations of the authors of these publications, we have built a custom test bed comprised of a threezone furnace, digital thermo-controller and turbo-molecular pump. Crystal growth takes place inside a quartz tube in the furnace under the constant flow of an inert gas. Starting materials are placed at the higher temperature furnace zone, and the creation of precisely controlled temperature gradient allows formation of crystals at the low temperature furnace zone. The main advantage of this setup is the precise control conditions maintained by the state-of-the-art precisely calibrated equipment. This test bed allows for very tight experimental conditions to be maintained for the growth of very unique crystals. Examples of crystals grown with this experimental technique will be presented.

\section{References:}

[1] R.A. Laudise, Ch. Kloc, P.G. Simpkins, T. Siegrist, Physical vapor growth of organic semiconductors, Journal of Crystal Growth 187 (1998) 449-454

[2] D. Vermeulen, L.Y. Zhu, K.P. Goetz, P. Hu, H. Jiang, Charge transport properties of Perylene-TCNQ crystals: The Effect of stoichiometry, Journal of Physical Chemistry C 118 (2014) 24688-24696

[3] O.D. Jurchescu, A. Meetsma, T. T. M. Palstra, Low-temperature structure of rubrene single crystals grown by vapor transport, Acta Cryst. (2006). B62, 330-334

Acta Cryst. (2018). A74, a311 